\title{
Mingle Face Detection using Adaptive Thresholding and Hybrid Median Filter
}

\author{
Amandeep Kaur \\ Department of Computer Science and Engg \\ Guru Nanak Dev University \\ Amritsar, India-143005
}

\begin{abstract}
Face detection has many applications in the field of digital image processing like face recognition, face analysis etc. Various techniques have been proposed so far to detect faces in images by different researchers. This paper presents a noble face detection algorithm which integrates the adaptive segmentation and hybrid median filter to improve the performance of face detection algorithms. Single faces noisy images are considered in this research work to evaluate the performance of the proposed algorithm. The results are compared with some well known face detection algorithms.
\end{abstract}

\section{Keywords}

face detection, adaptive thresholding, hybrid median filter, image processing, single face image

\section{INTRODUCTION}

Face detection deals with detecting faces in an image. It is complicated because of complex background, various illumination conditions, and large amount of facial expressions and so on. Therefore an effective approach is needed. Face detection is an important part of face recognition as the first step of automatic face recognition. It has numerous applications in areas like surveillance and security control systems, content-based image retrieval, biometrics recognition and multimedia applications, such as face tracking, face recognition, and video surveillance. Various government agencies are now more motivated to improve security data systems based on body or behavioral characteristics, often called biometrics. The most common biometrics are fingerprints and iris, but many other human characteristics have been studied in last years such as finger/palm geometry, voice, signature, face. UID Authority of India (UIDAI) has been setup by the Govt. of India with a mandate to issue a unique identification number to every resident in the country proposes to use biometrics for de-duplication and verification/authentication.

The face plays an important role in carrying identity of persons. Humans have ability to recognize the faces. Humans can recognize thousands of faces and identify familiar faces despite large changes in the visual stimulus due to viewing conditions, expression, aging, sex, and distractions such as glasses, or changes in hair .It is very useful for security purposes. Humans have the natural ability to recognize the face but developing a computer algorithm to do the same thing is difficult.

\section{RELATED WORK}

Chandrappa et al.[1] have proposed a novel technique for detecting faces in color images using skin color model algorithm combined with skin likely-hood, skin segmentation, Morphological operation and Template matching. This system achieved high detection accuracy, high detection speed and reduces the false detecting rate.
Chen et al. [2] have presented an automatic people counting system based on face detection, where the number of people passing through a gate or door is counted by setting a video camera. The basic idea is to first use the frame difference to detect the rough edges of moving people and then use the chromatic feature to locate the people face.

Kherchaoui et al. [3] has proposed an automatic system in which regions which are expected to contain human skin in the color image are detected. In the second step, faces are detected in the image by further analysis of skin areas by verifying a set of geometrical constraints related to common face characteristics and a template matching is applied to reach the final decision.

Kumar et al. [4] proposed algorithm initially estimates and projects the lighting compensated red component (LCRC) content of the image which is then subjected to skin detection algorithm which detects only the true skin regions in the image. The signatures corresponding to face candidates will be less than that of other candidates.

Liu et al .[5] has proposed a fast and self-adaptive face detection algorithm in which HSV color space whose intensity and chromaticity are separate and use only HS for detection. After that skin color detection is performed by using histogram back projection in which a binary image of skin color area was obtained after thresholding.

Pan et al. [6] presented a new face detection algorithm based on both face-color and hair-color space. For each skin color center point, to defect that whether there is a hair color center pixel in its right-top area. If there is a center point then stop the scanning, and regard the present skin color center pixel as the starting of the face detection.

Shehadeh et al. [7] proposed system which computes the difference between each vector in the reference matrix and pixels in the input image. If the difference is less than a threshold then this pixel is set to be skin. The circularity of the human faces was used as a feature to detect, localize and distinguish human faces form other objects.

Talele et al. [8] has given a method in which brightness normalization is performed using histogram equalization method. Adaboost is a boosting technique which is used to select a small no of features and to design strong classifier. For the extracted faces, orientation of face is measured based on the location of two eyes.

Kumar et al. [9] presented processes of image processing that are applied on images to remove the different types of noise that are either present in the image during capturing or introduced into the image during transmission. The one type of noise, salt \& pepper (impulse) which is occurred during transmission of the images or due to bit errors or dead pixels in the image contents. This paper deals with removing the impulse noise and blurredness simultaneously from the images. The hybrid filter is represented as the combination of wiener filter and median filter.

Mustafa et al. [10] gives a modified version of Hybrid Median filter, statistical filter for noise reduction. Statistical filter 
computes the median of the diagonal elements and the mean of the diagonal, horizontal and vertical elements in a moving window and finally the median value of the two values will be the new pixel value. The proposed method gives better results as compared to the classical implementation of the Mean, Median and Hybrid Median filter in terms of denoising quality.

\section{PROBLEM DEFINITION}

This method deals with detecting the human faces in input image and returning the locations of faces in images. Certain issues have to be measured while developing a face detection system that is not of much importance in other object detection problems. Illumination, facial hair, makeup, head pose and facial expression have to be kept in mind while evolving the face detection system. This requires a wellorganized method to characterize the face images.

This technique adopts the integration of adaptive thresholding and hybrid median filter will be used for face detection. Adaptive thresholding is used for image segmentation. Hybrid median filter is windowed filter of nonlinear class that easily removes impulse noise while preserving edges. Hybrid median filter one has better corner preserving characteristics in comparison with basic version of the median filter. The fundamental idea behind filter is that median technique is applied several times varying window shape for any element of the signal (image) and then take the median of the got median values.

The hybrid median filter is another modification of median filter. This filter is also called as corner preserving median filter is a three-step ranking operation. The median values of the $45^{\circ}$ neighbors forming an " $\mathrm{X}$ " and the $90^{\circ}$ neighbors forming a "+" are compared with the central pixel and the median value of that set is then saved as the new pixel value.

\section{RESEARCH METHODOLOGY}

In the proposed method, the goal is to detect the presence of faces in input images. We have concentrated on single face images in this method. It consists of 4 steps: Filtering using hybrid median filtering, Segmentation of adaptive thresholding, Minimization of background portion and detection of face objects. The proposed method is implemented using MatLab.

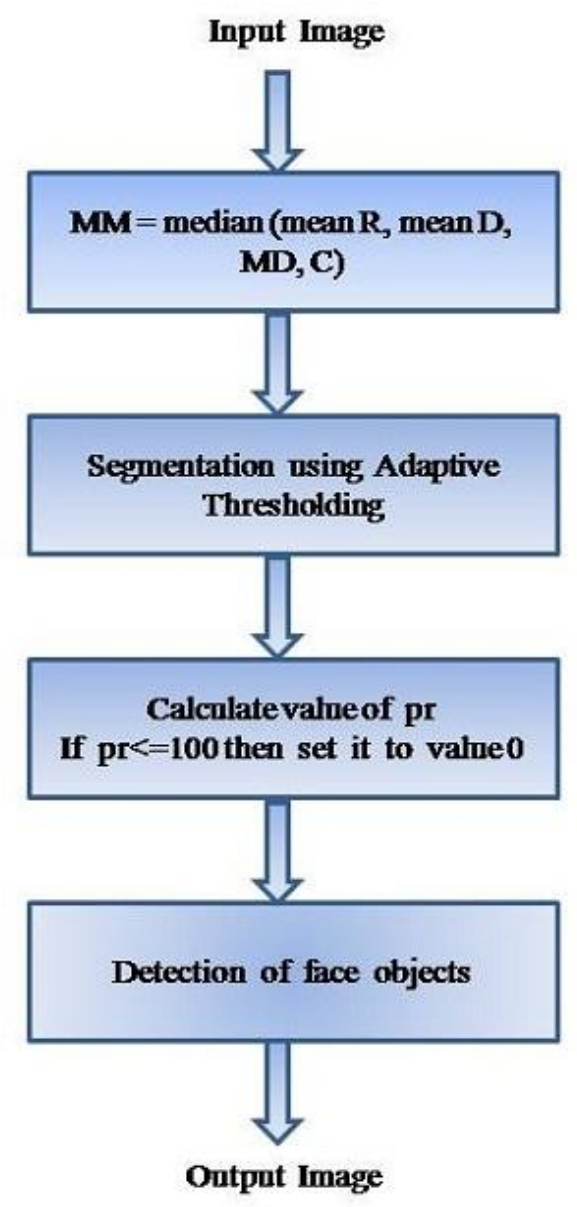

Fig 1: Research Methodology

Step 1: $M M=$ median (mean R, mean D, MD, C)

Mean $\mathrm{R}$ is the mean of horizontal and vertical $\mathrm{R}$ pixels, Mean $\mathrm{D}$ is the mean of diagonal D pixels, and MD is the median of diagonal $\mathrm{D}$ pixels. The filtered value is the median of the two mean values, median values, and the central pixel $\mathrm{C}$ : median ([Mean R, Mean D, MD, C]).

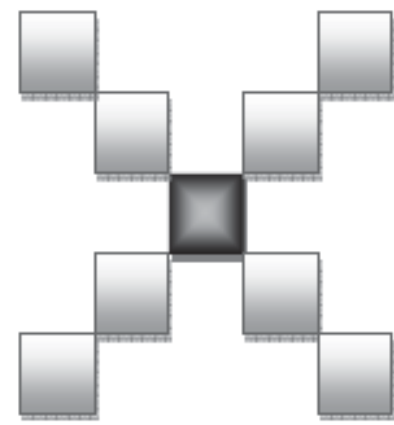

(a) 


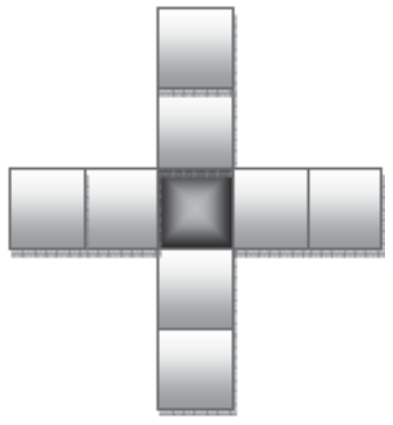

(b)

Fig 2: (a) Diagonal pixels (b) Horizontal and vertical pixels

Step 2: Segmentation of Adaptive Thresholding

Segmentation is a process that partitions an image into regions. Thresholding is used to segment an image by setting all the pixels whose intensity values are above a threshold to a foreground value and setting all the remaining pixels to a background value. Here a global threshold is used for all pixels and compared. But in an adaptive thresholding, the threshold dynamically changes over the image. This is a more sophisticated version of the thresholding and can accommodate changing lighting conditions in the image.

Step 3: Minimization of background portion

Value of pr is calculated.

If $\mathrm{pr}<=100$ then set it to value 0 .

Step 4: Detection of face objects.

\section{PERFORMANCE ANALYSIS}

\subsection{Hits}

It is defined as the no of faces detected in the experiment.

\subsection{Misses}

It is defined as the no of faces which are not detected in the experiment.

\subsection{Hit Ratio}

It is defined as no. of faces detected to the total no. of faces in the image.

\subsection{Miss Ratio}

$$
\text { Hit Ratio }=\frac{\text { No of faces detected }}{\text { Total no of faces in the image }}
$$

It is defined as no. of missed faces to the total no. of faces in the image

Miss Ratio $=1-$ Hit Ratio

\section{COMPARISON BETWEEN EXISTING METHOD AND PROPOSED METHOD}

Table 1. Features difference between Existing Method and Proposed Method

\begin{tabular}{|l|l|l|}
\hline Parameter & $\begin{array}{l}\text { Existing } \\
\text { Method }\end{array}$ & $\begin{array}{l}\text { Proposed } \\
\text { Method }\end{array}$ \\
\hline Filtering & No & Yes \\
\hline Overhead & More & Average \\
\hline Euler Test & Yes & No \\
\hline Template Matching & Yes & No \\
\hline Time Complexity & More & Less \\
\hline $\begin{array}{l}\text { Morphological } \\
\text { Operations }\end{array}$ & Yes & No \\
\hline Region Labelling & Yes & No \\
\hline
\end{tabular}

\subsection{Filtering}

Filtering is applied on images to remove the different types of noise that are either present in the image during capturing or introduced into the image during transmission.

\subsection{Overhead}

Overhead consists of extra computations that are to be performed to get the results. In the proposed method less computations are to performed as compared to that of existing method in which operations like Euler test, morphological operations, template matching etc, are also performed

\subsection{Euler Test}

The number of holes in each region to detect faces regions because face has more variance as compared to hands and arms.

\subsection{Template Matching}

At last cross correlation between template face and grayscale region is performed.

\subsection{Time Complexity}

It is defined as the time taken by the method to give results. Fewer computations are performed in the proposed method and hence it takes less time.

\subsection{Morphological Operations}

Morphology in image processing is a tool for extracting image components that are useful in the representation and description of region shape, such as boundaries and skeleton. Morphological operators are used to remove noisy regions and fill holes in the skin-color region.

\subsection{Region Labelling}

Region Labeling is labeling of clustered group of pixels so that can be identified as a single region, in order for each region to be analyzed further to determine if it is a face region or not. 


\section{RESULTS AND PERFORMANCE}

\section{ANALYSIS}

The experiment is performed by taking 94 images. The results show that the proposed method gives better results as compared to the existing method. The existing method gives 91 hits while the proposed method gives 92 hits. The existing method gives 3 misses and proposed method gives 2 misses. We have concentrated on single face images. The table illustrates face detection performance. As a result of this hit ratio of proposed method is more as compared to that of existing method.

Table 2. Existing Method Vs Proposed Method

\begin{tabular}{|l|l|l|}
\hline Parameter & Existing method & Proposed method \\
\hline Hits & 91 & 92 \\
\hline Misses & 3 & 2 \\
\hline Hit Ratio & 0.968 & 0.978 \\
\hline Miss Ratio & 0.032 & 0.022 \\
& & \\
\hline
\end{tabular}

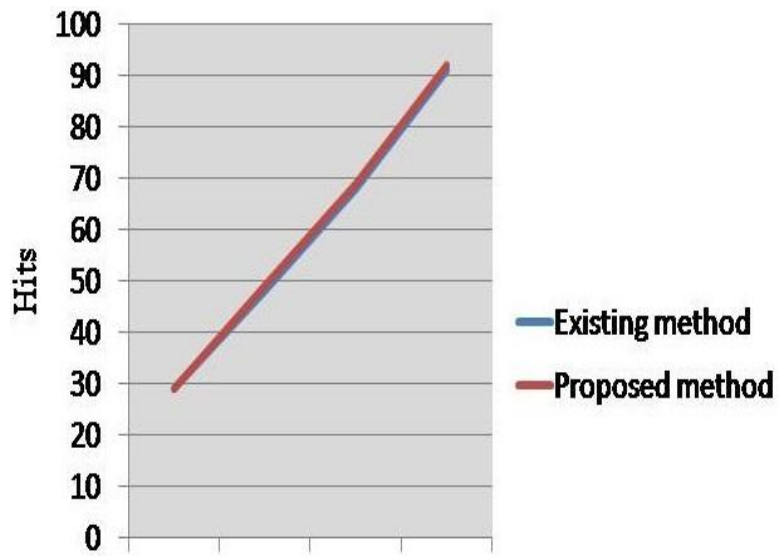

$\begin{array}{llll}30 & 50 & 70 & 94\end{array}$

No of faces

Fig 3: Hits vs. No. of faces

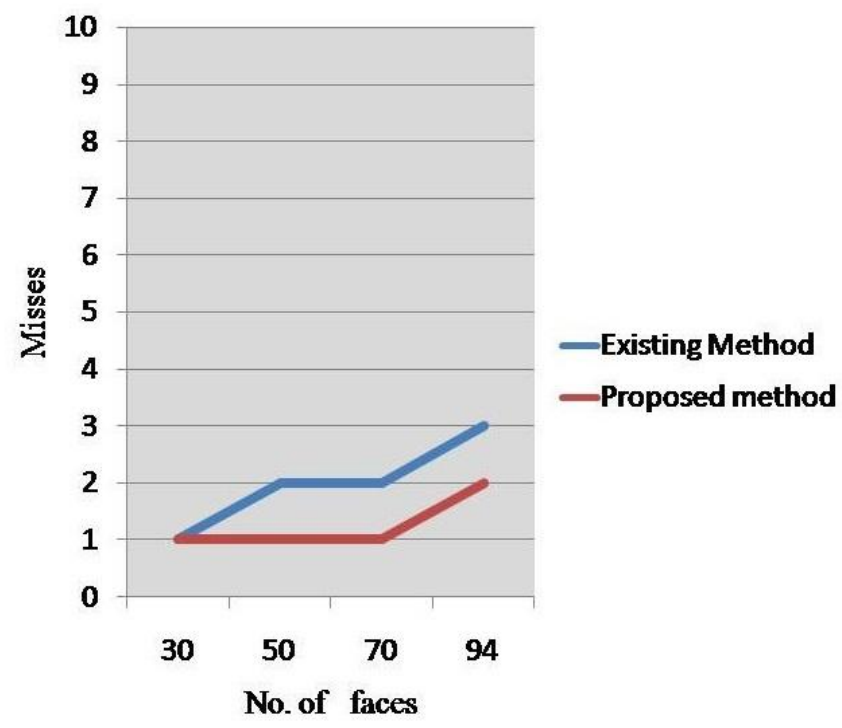

Fig 4: Misses vs. No. of faces

It is demonstrated in figure 3 there is a thin line of improvement in the no. of face detected i.e. hits in case of existing method

It is clear in figure 4 that no of faces not detected i.e. misses is same at the initial point for both existing and proposed method but after that there is a difference of one point between both as the proposed method show less no of misses.

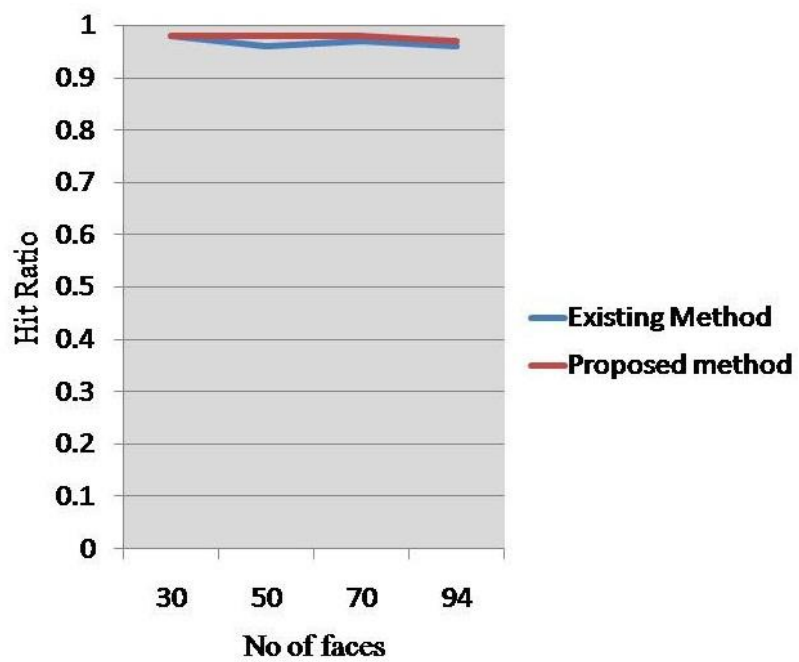

Fig 5: Hit Ratio vs. No. of faces

It is demonstrated in figure 5 that hit ratio of proposed method is more by 0.01 points as compared to existing method. 


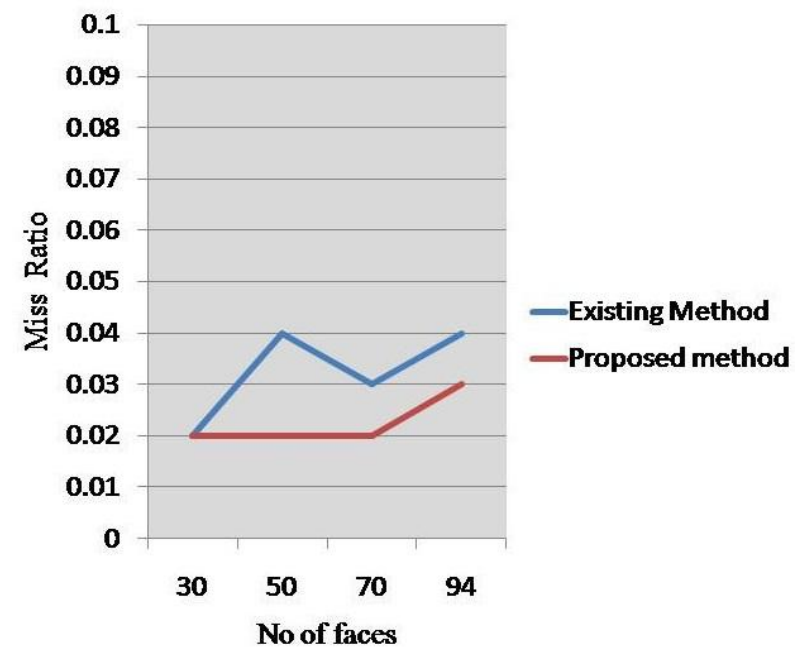

Fig 6: Miss Ratio vs. No of faces

It is clear in figure 6 that miss ratio of both methods is same at the initial point after that miss ratio of proposed method is 0.02 points less than that of existing method and after that the difference remained 0.01 .

\section{CONCLUSION}

It is shown that "mingle face detection using adaptive thresholding and hybrid median filter" algorithm provide better results than existing method. By considering suitable simulation it is proven that when noise is present in images, existing method most of the time fails to detect the faces. Therefore by using filtering we have achieved $97.8 \%$ efficiency. In the future work, this algorithm can be improved by using other filters for different type of noises on the images with more than one face.

\section{REFERENCES}

[1] Chandrappa D.N., Ravishankar M., RameshBabu D. R., "Face Detection in Color Images using Skin Color Model Algorithm based on Skin Color Information", 3rd International Conference on Electronics Computer Technology (ICECT), vol 1, page 254-258, IEEE, 2011.

[2] Chen T., Chen C., Wang D., Kuo Y., “ A People Counting System Based on Face-Detection", Fourth International Conference on Genetic and Evolutionary Computing, IEEE,2010.
[3] Kherchaoui S., Houacine A., "Face Detection Based On A Model Of The Skin Color With Constraints And Template Matching", International Conference on Machine and Web Intelligence (ICMWI), IEEE, 2010.

[4] Kumar C. N. R., Bindu A., "An Efficient Skin Illumination Compensation Model for Efficient Face Detection", 32nd Annual Conference on IEEE Industrial Electronics, 2006.

[5] Liu Q., Peng G., "A Robust Skin Color Based Face Detection Algorithm”, 2nd International Asia Conference on Informatics in Control, Automation and Robotics , IEEE, 2010

[6] Pan W., Huang Y., "A new algorithm of face detection under strong light condition", 2nd International Conference on Advanced Computer Control (ICACC), IEEE, 2010.

[7] Shehadeh H., Al-khalaf A., Al-khassaweneh M., "Human Face Detection Using Skin Color Information", IEEE International Conference on Electro/Information Technology (EIT), 2010.

[8] Talele K. T., Kadam S., "Face Detection and Geometric Face Normalization", TENCON, Singapore, IEEE Region 10 Conference, 2009.

[9] Kumar V., Priyanka, Kishore K., "A Hybrid Filter for Image Enhancement", International Journal of Image Processing and Vision Sciences (IJIPVS) Volume-1 Issue-1 ,2012.

[10] Mustafa Z. F., Abrahim B. A., Kadah Y. M., "K11 Modified Hybrid Median Filter for Image Denoising", $29^{\text {th }}$ National Radio Science Conference (NRSC 2012)April 10-12, 2012, Faculty of Engineering/Cairo University, Egypt. 